\title{
Data Processing And Experiment Of Barometric Altimeter Based On Recursive Least Squares Filtering
}

\author{
Xiaolei Wang, Shuangjian Yan, Linjiao Ren, Jitao Zhang, Xiaowan Zheng, Lingzhi Cao \\ College of Electric and Information Engineering, Zhengzhou University of Light Industry, Zhengzhou 450002, China
}

Keywords: barometric altimeter, data processing, recursive least squares filtering, height evaluation.

\begin{abstract}
It is an effective method to measure the height using a barometric altimeter, but there exists larger noise defect. In this paper, the height measurement system is designed by using the barometric pressure sensor, and the cause of noise is analyzed. In order to suppress the noise, the method of recursive least squares filtering is proposed to evaluate the measured height. A recursive least squares filter model is constructed and the recursive step is given, then the recursive method is applied to the height evaluation. An experiment is done to measure the floor height of a building, and the results show that the recursive least squares filtering method can make the evaluation data strictly track the original data change, the height measurement noise is suppressed effectively, and the height fluctuation reduces to $20 \%$ of the original measurement value.
\end{abstract}

\section{Introduction}

It is of very important significance to measure the height, which mainly used in the building height measurement, mountain or hillside height measurement, three-dimensional system navigation and other low range height measure systems. The height measurement methods include mechanical pressure altimeter, GPS height positioning ${ }^{[1-2]}$ and barometric altimeter ${ }^{[3]}$, etc., in which the barometric altimeter is widely popular because of its low cost, high precision, small size and other excellent characteristics ${ }^{[4]}$.

The method of measuring height using a barometer is based on the scientific evidence of atmospheric pressure variation with altitude, and the local test point altitude value or the height value relative to ground can be obtained accurately. However, due to the weather change, air flow conditions and other environmental factors, the barometric altitude measurement introduces some large measurement noise, which sometimes even results in unstable measure result. Therefore, some measure must be taken to suppress the noise. Commonly used methods are the first-order lag integral filtering $^{[5]}$ and polynomial filtering ${ }^{[6]}$. Based on the advantages of least squares method, in this article the recursive least squares method is provided to estimate the measurement height to ensure that the evaluation result is optimal in the square sense ${ }^{[7]}$.

\section{Barometric altimeter sensor and height measuring principle}

The core component of the barometric altitude measurement system is the atmospheric pressure sensor, which senses the atmospheric pressure change on the basis of the piezoresistive effect, and then calculates the height by the relationship between the pressure and the height. Pressure sensors include APM's TP015P, MOTO's MPX4115A and BOSCH's BMP085 and BMP180, etc., and TP015P and MPX4115A output analog signals, which must pass through signal conditioning and other operations to achieve the digitalization and the process is complex. BMP180 is the second generation of BMP085, which is of smaller noise and smaller size, outputs the digital signal directly, so it is simple in circuit and easy to use ${ }^{[8]}$.

The height measuring principle using the barometric sensor BMP 180 is constructed as shown in figure 1. As the CPU, the microprocessor controls and receives the sampled temperature and barometric pressure data, which is sensed by the BMP180 and processed into digital signal. Then the microprocessor compensates the pressure by the measure temperature and the preset calibration data, and calculates the height according to the relationship of atmospheric pressure and height. Finally, the concerned data is transmitted to LED screen to display through $\mathrm{I} 2 \mathrm{C}$ or SPI bus, or sent to PC through UART serial port protocol for further processing.

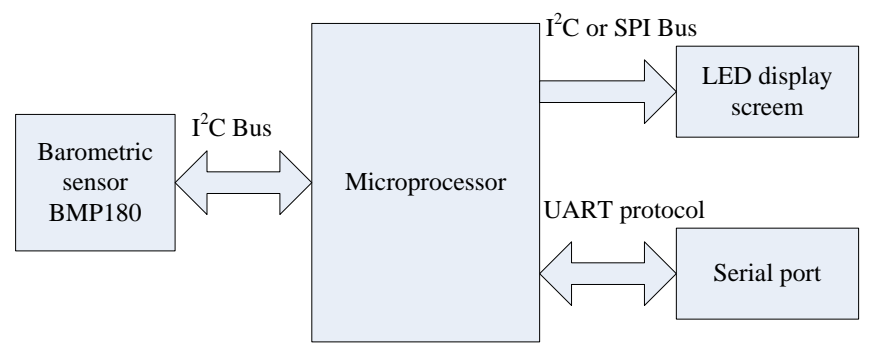

fig.1 Principle of height measuring using BMP180 
The numerical relationship of the measure pressure and the height is provided by the BMP180 datasheet, which is represented by the following formula (1).

$$
H=44330 \times\left[1-\left(\frac{P}{P_{0}}\right)^{1 / 5.255}\right]
$$

Which, $\mathrm{P}_{0}$ is the pressure at sea level, e.g. $1013.25 \mathrm{hPa}$ at $15^{\circ} \mathrm{C}$; $\mathrm{P}$ is the measured pressure in $\mathrm{hPa}$ and $\mathrm{H}$ is the altitude height in meter. Thus, a pressure change of $\Delta \mathrm{P}=1 \mathrm{hPa}$ corresponds to $8.43 \mathrm{~m}$ at sea level. The curse of barometric pressure and altitude above sea level is shown as figure 2 .

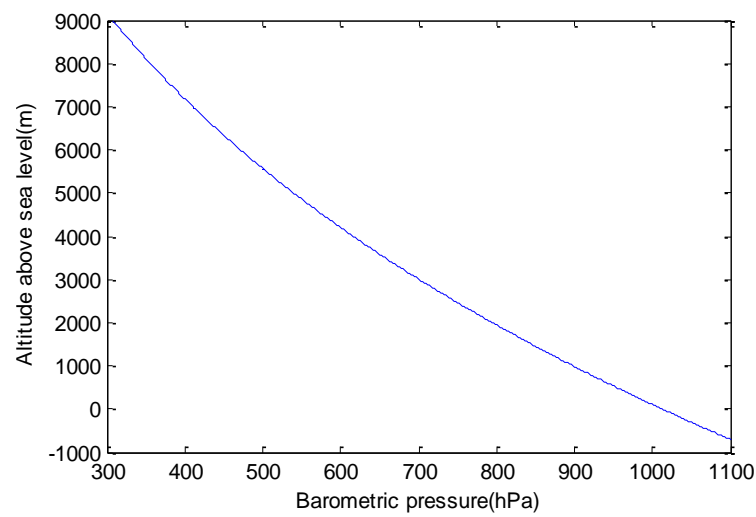

fig. 2 Curse of barometric pressure and altitude

According to the formula (1), the measure height relative to sea level can be calculated. Furturemore, the height relative to any ground plane can be obtained indirectly.

\section{Recursive least squares filter}

\subsection{Characters of recursive least squares filter}

Recursive least squares filtering method is on the basis of the least squares principle, using recursive method to obtain the optimal value, so that the sums of squares of the errors of the estimated values relative to the actual ones are minimized. The use of the recursive method avoids the problem of complicated calculation, poor real-time performance and even instability due to large amounts of data calculation.

Recursive least squares filtering estimation is a process of recursive and update parameters. When the system is running, each time the new observation data is acquired, based on the previous estimation result, the newly estimated observation data is used to revise the result of the previous estimation, so as to recursively obtain the new parameter estimation value. Unlike the minimum mean square estimation algorithm which has a fixed forgetting factor, the forgetting factor of the recursive least squares estimation is changed every time according to the recursive result. In addition, for the time variable barometric pressure, the height is also a time variable parameter, and the changed forgetting factor makes the recursive least squares estimation value still accurately track the height changes.
Therefore, the recursive least squares estimation method has a faster convergence speed, better stationary and signal adaptability.

\subsection{Construction of recursive least squares filter}

A set of M-order linear filters is constructed, as shown in Figure 3. The expected response $y(n)$ is estimated using linear combinations, which is

$$
\hat{y}(n)=\sum_{k=1}^{M} w_{k}(n) x_{k}(n)=\mathbf{w}^{T}(n) \mathbf{x}(n)
$$

Where, $w(n)$ is the weighting coefficient vector.

The estimation error $\mathrm{e}(\mathrm{n})$ is

$$
e(n)=y(n)-\hat{y}(n)=y(n)-\mathbf{w}^{T}(n) \mathbf{x}(n)
$$

The sum of squares of error e (n) is

$$
E=\sum_{n=0}^{N-1}[e(n)]^{2}
$$

For a linear time-invariant system, the coefficient vector remains constant throughout the whole measurement process.

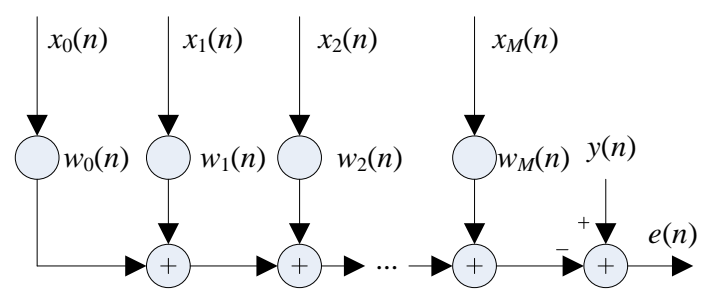

fig.3 M-order linear filter

Least Squares adaptive filtering estimation is a filter based on the least squares criterion, which minimizes the square error of the coefficients at each moment of filter operation. The cost function[9-10] is

$$
E(n)=\sum_{j=0}^{n} \lambda^{n-j}|e(j)|^{2}=\sum_{j=0}^{n} \lambda^{n-j}\left|y(j)-\mathbf{w}^{T}(j) \mathbf{x}(j)\right|^{2}
$$

Where, $\lambda$ is the forgetting factor, a positive constant of not more than 1 , which ensures that the filter can retain the most recent data and forget the past data, so that the algorithm is suitable for non-stationary environment. When $\lambda$ is 1 , the optimal criterion for minimizing $\mathrm{E}(\mathrm{n})$ make the least squares adaptive filter degenerate into the ordinary least squares method.

The autocorrelation matrix $R(n)$ of the input variable $x(j)$ is

$$
\mathbf{R}(n)=\sum_{j=0}^{n} \lambda^{n-j} \mathbf{x}(j) \mathbf{x}^{T}(j)
$$

By solving the cost function (5), the adaptive gain $\mathrm{g}(\mathrm{n})$ can be obtained.

$$
\mathbf{g}(n)=\frac{\lambda^{-1} \mathbf{P}(n-1) \mathbf{x}(n)}{1+\lambda^{-1} \mathbf{x}^{T}(n) \mathbf{P}^{T}(n-1) \mathbf{x}(n)}
$$

Where, $\mathrm{P}(\mathrm{n})$ is the inverse of the autocorrelation matrix $\mathrm{R}(\mathrm{n})$ and can be expressed as 


$$
\mathbf{P}(n)=\lambda^{-1} \mathbf{P}(n-1)-\lambda^{-1} \mathbf{g}(n) \mathbf{x}^{T}(n) \mathbf{P}^{T}(n-1)
$$

The weighting coefficient is

$$
\mathbf{w}(n)=\mathbf{w}(n-1)+\mathbf{g}(n) e(n)
$$

The above formulas(3), (6)-(9) constitute the recursive process of the recursive least squares evaluation filter. Under the rules, with the advancement of the data, the estimation value is constantly updated.

\subsection{Recursive least squares method applied in barometric height evaluation}

In views of the advantages of recursive least squares filtering, the recursive filtering method is used to the actual height evaluation.

For this purpose, $\mathrm{x}(\mathrm{n})$ is regarded as a set of standard reference values, and the height measured by the barometric altimeter is taken as the observation value $y(n)$, then $w(x)$ is the evaluated height value. Under the newly weight coefficients expressed by the forgetting factor, the different gain coefficient can be obtained, so that the evaluation value $\mathrm{w}(\mathrm{x})$ updates to the new value. By constantly recursive computation, the recursive least-squares filtering makes the evaluation result $\mathrm{w}(\mathrm{x})$ accurately track the change of $\mathrm{y}(\mathrm{x})$ and the noise is greatly suppressed when the square of the error is minimized.

\section{Experiment and analyzing}

In order to verify the effectiveness of the recursive least squares filtering method, a six floors of buildings in Zhengzhou City is chosen as the research object, and the selfmade barometric altimeter is used to measure the height of different floors in ascending order from low to high in sunny days. Through the continuous measurement, the original height value of each floor in $1 \sim 6$ floors is obtained. The altitude measurement is taken as the observation value, recursive least squares filtering method is used for loop iteration to evaluate the height, and the height evaluation value is obtained. Through the serial port and UART protocol, the height measurement and evaluation values are transmitted to the PC. The graphics are drawn that the evaluation data changes with the original data, as shown in figure 4 , where the sampling frequency of 5 points per second.

It can be seen from figure 4 that, after recursive least squares filtering, the evaluation value can strictly track the change of the measured height value, and the fluctuation of the evaluated height value is obviously reduced and the shape is not changed, which show that the recursive least-squares filtering can suppress the noise without distortion.

To measure the height of some a place for about one hour, and the original measurement data and the evaluation data can be obtained. The contrast curve of the original data and evaluation data is shown in figure 5 , and the height changes from $2.5 \mathrm{~m}$ to $0.5 \mathrm{~m}$ after filtering, which show that the estimated height fluctuation amplitude reduces to $20 \%$ of the original measurement value.

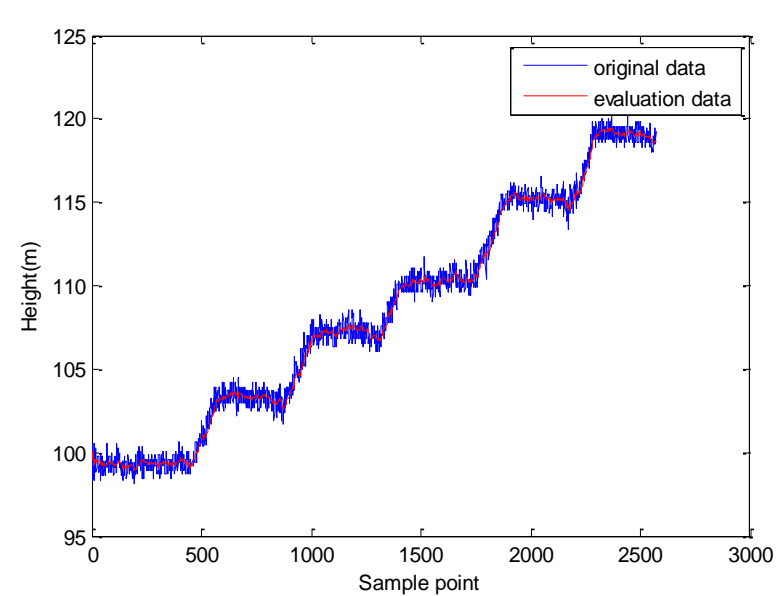

fig.4 Evaluation data changes with the original data

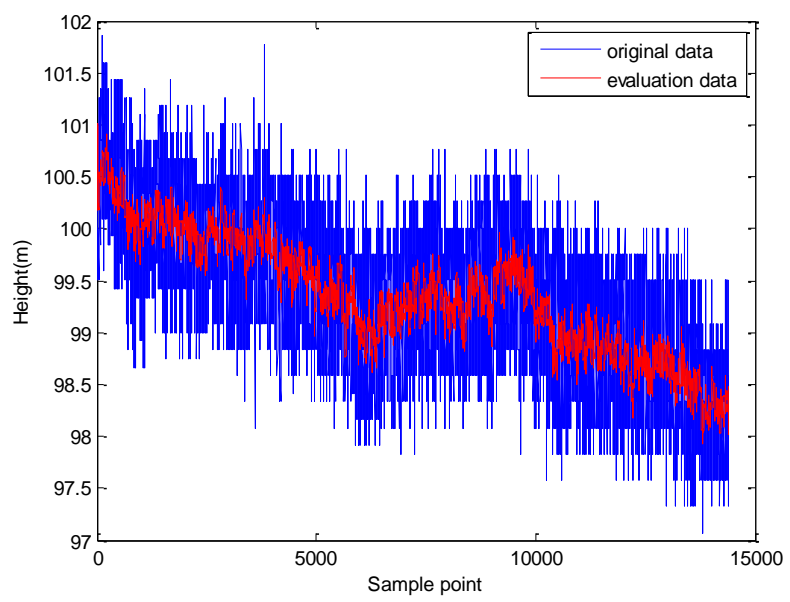

fig.5 Contrast curve of the original and evaluation data

It is worth noting that the height of the measurements is related to the atmospheric pressure at sea level. When the measured pot is far away from sea level, for the unknown sea level pressure the measured atmospheric pressure and the height may change at different weather. For example, in the case of cloudy days, the height is measured using the barometric altimeter in the same way. As the local weather changes causes the atmospheric pressure changes, but the sealevel pressure changes are unknown, if the height is still be calculated in accordance with the formula (1), the deviation is relatively large. However, if calculating the height relative to a local ground plane, the relative height value is still accurate.

\section{Conclusions}

In this paper, the methods commonly used to measure the height are analyzed, and the advantages of using atmospheric pressure measurement height is analyzed. According to the relationship between atmospheric pressure and altitude, a barometric pressure measurement system is designed. Aiming at the measurement noise caused by weather change and air flow velocity in the measuring environment, a recursive leastsquares filtering method is proposed to evaluate the height. The recursive least squares filtering model is constructed, and the recursive computation steps are given and the recursive 
method is applied to the height evaluation. The height of each floor of a building is measured, the results show that the recursive least squares filtering method can make the evaluation value strictly track the height change, suppress the measurement noise without distortion, and the height fluctuation reduces to $20 \%$ of the original measurement value.

\section{Acknowledgements}

This research was financially supported by the foundation of science and technology research project of Henan province (No.162102210210) and the Doctor scientific research fund of ZZULI (No.2014BSJJ046).

\section{References}

[1] Zaliva, Vadim, and Franz Franchetti. "Barometric and GPS altitude sensor fusion." 2014 IEEE International Conference on Acoustics, Speech and Signal Processing (ICASSP), pp.7525-7529, (2014).

[2] Zhang, Jieying, Edwan E, Zhou J, et al. "Performance investigation of barometer aided gps/mems-imu integration." 2012 IEEE/ION Position Location and Navigation Symposium (PLANS), pp.598-604, (2012).

[3] Zihajehzadeh, Shaghayegh, et al. "Integration of MEMS inertial and pressure sensors for vertical trajectory determination." IEEE Transactions on Instrumentation and Measurement, 64, pp.804-814, (2015).
[4] Singh, Kulwant, et al. "Fabrication of electron beam physical vapor deposited polysilicon piezoresistive MEMS pressure sensor." Sensors and Actuators A: Physical, 223, pp.151-158, (2015).

[5] WANG, Jun-cai, et al. "Design of a portable altitude measurement system based on BMP085." Transducer and Microsystem Technologies,30, pp.123-125, (2011).

[6] Dickow, Andreas, and Gregor Feiertag. "A framework for calibration of barometric MEMS pressure sensors." Procedia Engineering,87, pp.1350-1353(2014)

[7] Wang, Cheng, and Tao Tang. "Recursive least squares estimation algorithm applied to a class of linear-inparameters output error moving average systems." Applied Mathematics Letters, 29, pp.36-41, (2014).

[8] Jahangiri, A., et al. "Design and Deployment of a Portable Platform for Remote Urban Pollution Monitoring." Journal of Nuclear and Particle Physics, 5, pp.75-78, (2015).

[9] Hao, Shi Xin1 Li Chunlong1 Li. "Adaptive noise canceling system based on RLS algorithm." Electronic Measurement Technology, 3, pp.44-46, (2010).

[10] Wang, D. Q. "Least squares-based recursive and iterative estimation for output error moving average systems using data filtering." IET Control Theory \& Applications, 5, pp.1648-1657, (2011). 